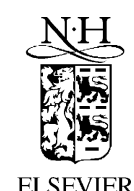

\title{
Dimensional reduction, magnetic flux strings, and domain walls
}

\author{
C.D. Fosco ${ }^{\text {a, } 1}$, A. López ${ }^{\text {a, } 1}$, F.A. Schaposnik ${ }^{\text {b,2 }}$ \\ a Centro Atómico Bariloche, 8400 Bariloche, Argentina \\ ${ }^{\mathrm{b}}$ Departamento de Física, Universidad Nacional de La Plata C.C. 67, 1900 La Plata, Argentina
}

Received 22 December 1999; accepted 22 May 2000

\begin{abstract}
We study some consequences of dimensionally reducing systems with massless fermions and Abelian gauge fields from $3+1$ to $2+1$ dimensions. We first consider fermions in the presence of an external Abelian gauge field. In the reduced theory, obtained by compactifying one of the coordinates "a la Kaluza-Klein", magnetic flux strings are mapped into domain wall defects. Fermionic zero modes, localized around the flux strings of the $3+1$ dimensional theory, become also zero modes in the reduced theory, via the Callan and Harvey mechanism, and are concentrated around the domain wall defects. We also study a dynamical model: massless $Q E D_{4}$, with fermions confined to a plane, deriving the effective action that describes the "planar" system. () 2000 Elsevier Science B.V. All rights reserved.
\end{abstract}

\section{Introduction}

Topological defects in quantum field theory (an excellent reference on the subject is [1]) play an important role in the description of many interesting phenomena, both in high energy and condensed matter physics applications. Being part of the non-perturbative spectrum, the understanding of their properties demands the study of topological classes of the field configuration space, and the realization of the associated topological invariants in the model under consideration. For some systems containing fermions, fermionic zero modes arise whenever the topological charge is different from zero. This effect has many important consequences for the study of the low momentum effective theory. Moreover,

\footnotetext{
${ }^{1}$ CONICET.

${ }^{2}$ Investigador CICBA, Argentina.
} 
since they are gapless excitations, they will strongly affect the response functions of the model [2].

In this paper we are concerned with the relationship between topological defects and zero modes in theories related by dimensional reduction. In particular, for the concrete example of fermions in $3+1$ dimensions coupled to an Abelian gauge field, we shall derive the low-momentum effective action for the dimensionally reduced theory. Being originally a massless theory, the momentum scale will be fixed by the inverse of the compactification length. Also, as it will be explained, quantum corrections coming from all the KaluzaKlein modes have to be included, to be consistent with large gauge invariance.

We will first consider the case of massless Dirac fermions in the presence of an external Abelian field. We then go on to consider a dynamical model: massless $Q E D$ in $3+1$ dimensions. The reduction is here introduced for the fermions, assumed to be confined to a planar spatial region. Being the only sources of the gauge field, this induces a reduction also for the gauge field, regardless of the fact that it is apriori unconfined. For this system we derive the effective theory, showing that there is room for the existence of "stripe" defects, i.e., localized zero modes that appear due to changes in the sign of the fermionic mass. This is just a manifestation of the well known Callan and Harvey mechanism [3]. When there is translation invariance along one of the spatial directions, the dynamics of the defects is governed by a sine-Gordon-like action. Moreover, the soliton charge is linked to the number of zero modes.

The organization of this paper is as follows: in Section 2, we consider fermions in the presence of an external (non-dynamical) gauge field, in $3+1$ dimensions. To take into account quantum effects, Section 3 deals with the evaluation of the effective action using a derivative expansion approximation for the fermionic determinant. In Section 4, we derive the low-momentum effective theory for the interacting case of massless $Q E D_{4}$. Finally, Section 5 is devoted to the conclusions.

\section{2. "Non-interacting" case}

By "non-interacting" we refer to a model containing a fermionic field $\psi$ in the presence of an external (i.e., non-dynamical) gauge field $A_{\alpha}$. The Euclidean action for the present case is simply defined by

$$
S_{F}=\int \mathrm{d}^{4} x \bar{\psi} \not D \psi
$$

where

$$
\not D=\gamma_{\alpha} D_{\alpha}, \quad D_{\alpha}=\partial_{\alpha}+\mathrm{i} e A_{\alpha} .
$$

We shall adopt the convention that indices from the beginning of the Greek alphabet always run over the values $0,1,2,3$ while those from the end belong to the set $0,1,2$ and are reserved for the coordinates of the reduced spacetime. The third spatial coordinate, $x_{3}$, is compactified: $0 \leqslant x_{3} \leqslant L$, and shall be sometimes also denoted ' $s$ ', to emphasize its special role, as opposed to the uncompactified coordinates, $x_{\mu}$. 
We shall first be concerned with configurations corresponding to magnetic flux strings in $3+1$ dimensions, with the strings entirely contained in the reduced hyperplane $x_{3}=0 .{ }^{3}$ These configurations are here considered regardless of the mechanism which may create them. To have these magnetic field structures, special gauge field configurations are required. Beforehand, we will use part of the gauge freedom to restrict the external gauge field configurations to the ones satisfying the condition:

$$
\partial_{3} A_{3}=0,
$$

which can always be fulfilled, by performing a regular gauge transformation connected to the identity. With this choice, $A_{3}(x, s) \rightarrow \tilde{A}_{3}(x)$,

$$
\tilde{A}_{3}(x) \equiv \frac{1}{L} \int_{0}^{L} \mathrm{~d} s A_{s}(x, s) .
$$

Besides considering static magnetic flux strings, we would also like to include a "small" gauge field configuration in order to find the effective action. The strings, being topological objects, should be treated non-perturbatively, while the small part is assumed to be intrinsically perturbative.

In $3+1$ dimensions, the magnetic field components $B_{i}(i=1,2,3)$ are, as usual, defined by $B_{i}=\epsilon_{i j k} \partial_{j} A_{k}$. As the flux strings lay on the $x_{3}=0$ plane, the third normal component of the magnetic field vanishes: $B_{3}=0$. Moreover, we can generate the two non-vanishing components $B_{1}, B_{2}$ using a gauge field such that $A_{1}=A_{2}=0$, and $\tilde{A}_{3} \neq 0$. With this choice,

$$
B_{i}(x)=\epsilon_{i j} \partial_{j} \tilde{A}_{3}(x) .
$$

It is evident that, from the point of view of the reduced, planar theory, $\tilde{A}_{3}$ is a scalar field under transformations mapping the plane onto itself. It is convenient to use the definition $\tilde{A}_{3}(x)=\varphi(x)$, with $x \equiv\left(x_{0}, x_{1}, x_{2}\right)$, to make explicit the fact that $\tilde{A}_{3}$ behaves like a scalar.

In the case of a flux string along the $x_{1}$ axis, the magnetic field is:

$$
B_{1}=\xi \delta\left(x_{3}\right) \delta\left(x_{2}\right),
$$

where $\xi$ is a constant. ${ }^{4}$

We need to write now a gauge field configuration leading to the magnetic field of (6). There are of course many possibilities but, interestingly enough, if we want to dimensionally reduce the theory, this freedom is substantially reduced. The reason is that we do not want a gauge field configuration having a nontrivial $x_{3}$ dependence far away from the $x_{3}=0$ hyperplane. The natural choice is then a gauge field strongly concentrated on $x_{3}=0$, and this determines the gauge field configuration to be of the form:

$$
A_{3}(x)=\frac{\xi}{2} \delta\left(x_{3}\right) \operatorname{sign}\left(x_{2}\right),
$$

\footnotetext{
${ }^{3}$ This is rather different to the more standard situation of a flux tube pointing in the $x_{3}$ direction, and thus corresponding to a magnetic vortex in $2+1$ dimensions.

${ }^{4}$ This $\delta$-like configuration is, of course, an idealization. Realistic configurations will be, although highly concentrated, regular functions.
} 
which implies, for the $2+1$ dimensional scalar field

$$
\varphi(x)=\frac{\xi}{2 L} \operatorname{sign}\left(x_{2}\right) .
$$

The constant $\xi$ is, of course, related to the (quantized) total flux of the string, thus $\xi=$ $2 \pi k / e$, where $k$ is an integer, counting the number of elementary fluxes.

We note the emergence of a step-like configuration for the scalar field. This scalar field, as we shall see next, plays the role of the mass for $2+1$ dimensional fermions. Hence, invoking the Callan and Harvey mechanism [3], we see that there are zero modes concentrated on the walls, which indeed correspond to the dimensionally reduced version of the zero modes around the flux strings. It is worth noting that the scalar field profile is discontinuous because of the assumed $\delta$-like profile for the magnetic field. It is convenient to use instead a regular profile,

$$
\varphi(x)=\frac{\xi}{2 L} h_{\Delta}\left(x_{2}\right)
$$

where $h_{\Delta}\left(x_{2}\right)$ is a smoothed version of the step function, for example,

$$
h_{\Delta}\left(x_{2}\right)=\tanh \left(x_{2} / \Delta\right),
$$

where $\Delta$ is a length which essentially measures the "width" of the stripe.

To discuss issues related to the fermionic zero modes, like the relationship between the number of modes in $3+1$ and $2+1$ dimensions, a more detailed study of the fermionic action (1) is required. The full gauge field configuration (perturbative plus nonperturbative) is assumed to be $s$-independent. This simplifying assumption allows us to take advantage of the fact that the gauge field $\tilde{A}_{3}$ is independent of $s=x_{3}$ to Fourier expand the fermionic fields:

$$
\begin{aligned}
& \psi\left(x, x_{3}\right)=\frac{1}{\sqrt{L}} \sum_{n=-\infty}^{+\infty} \mathrm{e}^{\mathrm{i} \omega_{n} x_{3}} \psi_{n}(x), \\
& \bar{\psi}\left(x, x_{3}\right)=\frac{1}{\sqrt{L}} \sum_{n=-\infty}^{+\infty} \mathrm{e}^{-\mathrm{i} \omega_{n} x_{3}} \bar{\psi}_{n}(x),
\end{aligned}
$$

with $\omega_{n}=2 \pi n / L$. We then obtain for $S_{F}$ a representation as a series of decoupled $2+1$ dimensional actions

$$
S_{F}=\sum_{n=-\infty}^{+\infty} S_{F, n} .
$$

The explicit form for the action $S_{F, n}$ corresponding to each Fourier mode is:

$$
S_{F, n}=\int \mathrm{d}^{3} x \bar{\psi}_{n}(x)\left[\gamma_{\mu} D_{\mu}+\mathrm{i} \gamma_{3}\left(\omega_{n}+e \varphi\right)\right] \psi_{n}(x) .
$$

We assume the Dirac matrices are in the representation:

$$
\gamma_{0}=\left(\begin{array}{cc}
0 & \sigma_{3} \\
\sigma_{3} & 0
\end{array}\right), \quad \gamma_{1}=\left(\begin{array}{cc}
0 & \sigma_{1} \\
\sigma_{1} & 0
\end{array}\right),
$$




$$
\gamma_{2}=\left(\begin{array}{cc}
0 & \sigma_{2} \\
\sigma_{2} & 0
\end{array}\right), \quad \gamma_{3}=\left(\begin{array}{cc}
0 & \mathrm{i} I \\
-\mathrm{i} I & 0
\end{array}\right)
$$

where $\sigma_{j}$, with $j=1,2,3$, denote the usual Pauli matrices, and $I$ is the $2 \times 2$ identity matrix. Writing each four-component fermionic field $\psi_{n}$ in terms of two 2-component fermions $\chi_{n}^{(a)}(a=1,2)$, we see that

$$
S_{F, n}=\int \mathrm{d}^{3} x\left[\bar{\chi}_{n}^{(1)}\left(d+\omega_{n}+e \varphi\right) \chi_{n}^{(1)}+\bar{\chi}_{n}^{(2)}(x)\left(d-\omega_{n}-e \varphi\right) \chi_{n}^{(2)}(x)\right] .
$$

We have introduced the notation $d$ to refer to the Dirac operator in $2+1$ dimensions, acting on 2-component fermions. More explicitly,

$$
d=\sigma_{\mu} D_{\mu}
$$

with $\sigma_{0} \equiv \sigma_{3}$, and $\sigma_{j}(j=1,2)$ again denoting the usual Pauli matrices. The covariant derivative depends on a gauge field which, in our approximation, is a function of $x_{\mu}$ only.

It is important to realize that all the fermionic modes should be kept if large gauge invariance is to be preserved, since large gauge invariance amounts to shifts in $\varphi$, and this field is directly coupled to the fermionic field.

It is now easy to study the Callan and Harvey fermionic zero modes of the reduced theory, assuming that in the original model in $3+1$ dimensions the background produced a non-vanishing, quantized, magnetic flux. We first realize that the decomposition (12) shows that there is an infinite number of $2+1$ dimensional Dirac fermions, each Fourier mode consisting of two flavours, distinguished by the sign of their mass terms. A Callan and Harvey zero mode for the Fourier component $n$ shall appear whenever $\omega_{n}+e \varphi(x)$ crosses zero more or less sharply. Thus, the configuration (8) (in its smooth version (9)) shows that there is at least a zero mode for the $n=0$ component, but, if there is a flux string with charge $k$, the scalar field profile will cross as many values of $\omega_{n}$, implying that there are, indeed, $k$ zero modes in the reduced theory. To see this, we take into account the expression (9) for the domain wall configuration. The equation determining the locii of the zero modes may be written as

$$
h_{\Delta}\left(x_{2}\right)=\frac{2}{k} \times \text { integer, }
$$

where $k$ is the (fixed) number of elementary fluxes. In Fig. 1 we show, as an example, the case of a stripe configuration $h_{\Delta}$ with $k=9$. The horizontal lines correspond to different $n$ 's, and a zero mode is produced whenever one of these lines intersects the curve.

It is interesting to realize that a proper understanding of the relation between the height of the stripe and the number of zero modes requires the use of the "smoothed" version of the steplike scalar field configuration. This should be evident from Fig. 1, if one imagines the curve to be deformed to a steplike configuration.

We remark that the zero modes produced in this way are massless Dirac fermions in $1+1$ dimensions, because each flavour produces a given chirality, and the signs are opposite. 




Fig. 1. A stripe configuration of charge 9.

\section{Derivative expansion for the fermionic determinant}

We shall now consider the evaluation of the fermionic determinant, as a functional of $a_{\mu}$ and $\varphi$, in a derivative expansion approximation. The field $a_{\mu}$ denotes the perturbative part of the gauge field.

To avoid any risk of confusion, we shall, from now on, use two-component fermions only. The "effective action" $\Gamma_{F}\left[\varphi, a_{\mu}\right]$ is defined as the result of functionally integrating out the fermionic fields. Using the Fourier decomposition for the $x_{3}=s$ coordinate, we see that

$$
\begin{aligned}
\mathrm{e}^{-\Gamma_{F}\left[\varphi, a_{\mu}\right]}= & \int \prod_{n=-\infty}^{+\infty} \mathcal{D} \bar{\chi}_{n}^{(1)} \mathcal{D} \chi_{n}^{(1)} \mathcal{D} \bar{\chi}_{n}^{(2)} \mathcal{D} \chi_{n}^{(2)} \\
& \times \exp \left\{-\sum_{n=-\infty}^{+\infty}\left[\bar{\chi}_{n}^{(1)}\left(d+\omega_{n}+e \varphi\right) \chi_{n}^{(1)}+\bar{\chi}_{n}^{(2)}\left(d-\omega_{n}-e \varphi\right) \chi_{n}^{(2)}\right]\right\} .
\end{aligned}
$$

Thus $\Gamma_{F}$ may be written in terms of fermionic determinants in $2+1$ dimensions:

$$
\begin{aligned}
\Gamma_{F}\left[\varphi, a_{\mu}\right] & =-\sum_{n=-\infty}^{+\infty}\left\{\ln \operatorname{det}\left(d+\omega_{n}+e \varphi\right)+\ln \operatorname{det}\left(d-\omega_{n}-e \varphi\right)\right\} \\
& =-\sum_{n=-\infty}^{+\infty} \operatorname{Tr} \ln \left[-d^{2}+\left(\omega_{n}+e \varphi\right)^{2}\right] .
\end{aligned}
$$

Large gauge transformations wind up a number of times around the periodic coordinate, $s$. Then they correspond to constant shifts in the scalar field $\varphi$ :

$$
\varphi(x) \rightarrow \varphi(x)+\frac{2 \pi n}{e L},
$$

where $n$ is an integer. It is now clear, from the general expression (19), that all the Fourier modes must be kept, if invariance under (20) is to be maintained. 
In the derivative expansion technique, which we shall use in order to evaluate $\Gamma_{F}$, the leading, zero derivative term, can usually be treated exactly. This term may be thought of as depending basically on the constant component of the fields. The following terms in the derivative expansion depend of course also on the fluctuating part, which is assumed to be small in comparison with the constant part.

The piece of the effective action depending on the constant part is usually regarded as an "effective potential". In our case, there is no point in keeping a constant part for $a_{\mu}$ since, if present, it could be gauged away (because the "planar" system is assumed to have a trivial topology). From (20), it is evident that to keep a constant component for $\varphi$ is, in turn, crucial. We thus decompose $\varphi$ into two pieces,

$$
\varphi(x)=\varphi_{0}+\tilde{\varphi}(x),
$$

where the constant $\varphi_{0}=\langle\varphi(x)\rangle$ denotes the $\left(x_{\mu}\right)$ spacetime average of $\varphi(x)$, and $\tilde{\varphi}(x)=$ $\varphi(x)-\varphi_{0}$. Then we factorize a constant field determinant,

$$
\Gamma_{F}\left[\varphi, a_{\mu}\right]=\Gamma_{F}\left[\varphi_{0}, 0\right]+\Delta \Gamma_{F}\left[\tilde{\varphi}, a_{\mu}\right]
$$

where

$$
\Gamma_{F}\left[\varphi_{0}, 0\right] \equiv \int \mathrm{d}^{3} x V_{\mathrm{eff}}(\varphi)=-\sum_{n=-\infty}^{+\infty} \operatorname{Tr} \ln \left[-\not \partial^{2}+\left(\omega_{n}+e \varphi_{0}\right)^{2}\right]
$$

and

$$
\begin{aligned}
\Delta \Gamma\left[\tilde{\varphi}, a_{\mu}\right]= & -\sum_{n=-\infty}^{+\infty} \operatorname{Tr} \ln \left[1+e\left(\not \partial+\omega_{n}+e \varphi_{0}\right)^{-1}(\mathrm{i} \not \not h+\tilde{\varphi})\right] \\
& -\sum_{n=-\infty}^{+\infty} \operatorname{Tr} \ln \left[1+e\left(\not \partial-\omega_{n}-e \varphi_{0}\right)^{-1}(\mathrm{i} \not h-\tilde{\varphi})\right] .
\end{aligned}
$$

We have introduced the notation ' $V_{\text {eff' }}$ ' under the integral symbol in (23), to emphasize the property that it will play the role of an effective potential for $\varphi$. At the end of the evaluation, we shall follow the common practice of replacing $\varphi_{0}$ by $\varphi$ in $V_{\text {eff. }}$ This approximation is justified within the derivative expansion technique, as long as the hypothesis leading to that expansion are valid [4,5].

Large gauge invariance means that this potential is a periodic function of $\varphi$. The constant field determinant is evaluated by taking the functional and Dirac traces in momentum space:

$$
\begin{aligned}
\Gamma_{F}\left[\varphi_{0}, 0\right] & =-\sum_{n=-\infty}^{+\infty} \operatorname{Tr} \ln \left[-\not^{2}+\left(\omega_{n}+e \varphi_{0}\right)^{2}\right] \\
& =-2 \int \mathrm{d}^{3} x \sum_{n=-\infty}^{+\infty} \int \frac{\mathrm{d}^{3} p}{(2 \pi)^{3}} \ln \left[p^{2}+\left(\omega_{n}+e \varphi_{0}\right)^{2}\right] .
\end{aligned}
$$

To evaluate the sum over $n$, we follow techniques of standard application in finite temperature quantum field theory [6]. We first take advantage of the fact that the series runs from $n=-\infty$ to $n=+\infty$, to write 


$$
\begin{aligned}
\Gamma_{F}\left[\varphi_{0}, 0\right]= & -\int \mathrm{d}^{3} x \sum_{n=-\infty}^{+\infty} \int \frac{\mathrm{d}^{3} p}{(2 \pi)^{3}} \\
& \times\left\{\ln \left[p^{2}+\left(\omega_{n}+e \varphi_{0}\right)^{2}\right]+\ln \left[p^{2}+\left(\omega_{n}-e \varphi_{0}\right)^{2}\right]\right\},
\end{aligned}
$$

which is easily rearranged as

$$
\begin{aligned}
\Gamma_{F}\left[\varphi_{0}, 0\right]= & -\int \mathrm{d}^{3} x \sum_{n=-\infty}^{+\infty} \int \frac{\mathrm{d}^{3} p}{(2 \pi)^{3}} \\
& \times\left\{\ln \left[\omega_{n}^{2}+\left(p+\mathrm{i} e \varphi_{0}\right)^{2}\right]+\ln \left[\omega_{n}^{2}+\left(p-\mathrm{i} e \varphi_{0}\right)^{2}\right]\right\} .
\end{aligned}
$$

Each one of the terms in (27) may be evaluated by translating known results about the free energy for a system of free bosons [6], what yields,

$$
\Gamma_{F}\left[\varphi_{0}, 0\right]=-\int \mathrm{d}^{3} x \int \frac{\mathrm{d}^{3} p}{(2 \pi)^{3}}\left\{\ln \left[1-\mathrm{e}^{-L\left(p+\mathrm{i} e \varphi_{0}\right)}\right]+\ln \left[1-\mathrm{e}^{-L\left(p-\mathrm{i} e \varphi_{0}\right.}\right]\right\},
$$

where we have ignored the "zero point" contribution, since it is a $\varphi$-independent constant. We shall, however, fix the ambiguity in the renormalization of this infinite constant by

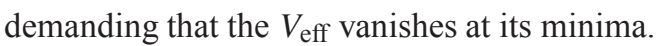

The integral over $p$ can be performed, and the result can be presented as a series,

$$
\Gamma_{F}\left[\varphi_{0}, 0\right]=\int \mathrm{d}^{3} x \frac{4}{\pi^{2} L^{3}} \sum_{n=-\infty}^{\infty} \frac{1}{n^{4}}\left[\cos \left(n e L \varphi_{0}\right)-(-1)^{n}\right],
$$

or:

$$
\int \mathrm{d}^{3} x V_{\text {eff }}=\int \mathrm{d}^{3} x\left[\frac{4}{\pi^{2} L^{3}} \sum_{n=-\infty}^{\infty} \frac{1}{n^{4}} \cos \left(n e L \varphi_{0}\right)-\frac{14 \pi^{2}}{5 L^{3}}\right] .
$$

Let us next consider the remaining part of the effective action, denoted $\Delta \Gamma_{F}[\tilde{\varphi}, a]$. Besides a dependence on $\varphi_{0}$, it will also depend on $\tilde{\varphi}$ (the fluctuating part of $\varphi$ ) and $a_{\mu}$.

To be consistent with the derivative expansion, and also because both $\varphi$ and $a_{\mu}$ are proportional to the same coupling constant $e$, the expansion must treat both fields as a single entity. ${ }^{5}$ To this end, we have found illuminating to define a new vector field $\sigma_{\mu}$, which summarizes the information on the $a_{\mu}$ and $\tilde{\varphi}$ configurations, through the relations

$$
\begin{aligned}
\epsilon_{\mu \nu \lambda} \partial_{\nu} \sigma_{\lambda} & =e a_{\mu}, \\
\partial_{\mu} \sigma_{\mu} & =e \tilde{\varphi}(x) .
\end{aligned}
$$

Of course, this involves the assumption that the Lorentz gauge $\partial \cdot a=0$ has been adopted for the gauge field. The number of independent components for $\sigma_{\mu}$ is three, since this field is, in principle, not constrained by any gauge invariance requirement, and this matches the number of components for the transverse $a_{\mu}$ (two) plus the scalar fields (one). Using trivial properties of the $\gamma$-matrices in $2+1$ dimensions, we observe that $\Delta \Gamma_{F}(\tilde{\varphi}, a)$ may be written as

\footnotetext{
${ }^{5}$ Indeed, they originate in different components of the same $3+1$ dimensional gauge field $A_{\alpha}$.
} 


$$
\Delta \Gamma_{F}\left[\tilde{\varphi}, a_{\mu}\right]=-\sum_{n=-\infty}^{\infty}\left\{\operatorname{Tr} \ln \left[1+\left(\not \partial+\omega_{n}+e \varphi_{0}\right)^{-1}(\not \partial \phi)\right]+\varphi \leftrightarrow-\varphi\right\} .
$$

Because of its explicit Lorentz covariance, this expression could be regarded as a consistency check for the procedure of introducing $\sigma_{\mu}$ as a Lorentz vector.

Taking into account the fact that (32) is explicitly even under parity transformations, we may write

$$
\Delta \Gamma_{F}\left[\varphi, a_{\mu}\right]=\Delta \Gamma_{\text {even }}\left[\partial_{\mu} \varphi-\tilde{F}_{\mu}\right]
$$

where the 'even' label refers to the behaviour under parity transformations, and $\tilde{F}_{\mu}=$ $\epsilon_{\mu \nu \lambda} \partial_{\nu} a_{\lambda}$. The particular dependence of $\Delta \Gamma_{F}$ on its arguments is due to the fact that:

$$
\sigma_{\mu}=e \frac{1}{\partial^{2}}\left(\partial_{\mu} \varphi-\tilde{F}_{\mu}\right)
$$

We calculate the leading term in a derivative expansion for $\Delta \Gamma_{F}$, by noting that, expanding up to second order in derivatives the $2+1$ dimensional object

$$
\gamma\left[\sigma_{\mu}\right]=\operatorname{Tr} \ln \left[1+\left(\not \partial+\omega_{n}+e \varphi_{0}\right)^{-1}(\not \partial \phi)\right],
$$

we obtain

$$
\gamma\left[\sigma_{\mu}\right]=\frac{e^{2}}{24 \pi\left|\omega_{n}+e \varphi_{0}\right|} \int \mathrm{d}^{3} x\left[\left(\partial_{\mu} \varphi-\tilde{F}_{\mu}\right)\left(\partial_{\mu} \varphi-\tilde{F}_{\mu}\right)\right] .
$$

Then we easily see that

$$
\begin{aligned}
\Delta \Gamma_{F}\left[\varphi, a_{\mu}\right] & =\left\{\gamma\left[\sigma_{\mu}\right]+(\varphi \leftrightarrow-\varphi)\right\} \\
& =\frac{e^{2}}{12 \pi} \int \mathrm{d}^{3} x \sum_{n=-\infty}^{\infty} \frac{1}{\left|\omega_{n}+e \varphi_{0}\right|}\left[\left(\partial_{\mu} \varphi\right)^{2}+\left(\tilde{F}_{\mu}\right)^{2}\right] .
\end{aligned}
$$

Namely, it corresponds to a local Maxwell term for $a_{\mu}$ and a local kinetic term for $\varphi$.

In summary, the effective action induced by the integration of the fermionic degrees of freedom is, to second order in a derivative expansion:

$$
\begin{aligned}
\Gamma_{F}\left[\varphi, a_{\mu}\right]= & \int \mathrm{d}^{3} x\left[\frac{4}{\pi^{2} L^{3}} \sum_{n=-\infty}^{\infty} \frac{1}{n^{4}} \cos (n e L \varphi)-\frac{14 \pi^{2}}{5 L^{3}}\right] \\
& +\frac{e^{2}}{12 \pi} \int \mathrm{d}^{3} x \sum_{n=-\infty}^{\infty} \frac{1}{\left|\omega_{n}+e \varphi\right|}\left[\left(\partial_{\mu} \varphi\right)^{2}+\left(\tilde{F}_{\mu}\right)^{2}\right],
\end{aligned}
$$

where we have replaced $\varphi_{0}$ by $\varphi$ in the contributions from the effective potential (30) and the subleading term in the derivative expansion of the fermionic determinant (37). As already mentioned, this procedure is justified within the derivative expansion approximation.

\section{Massless $Q E D_{4}$}

In previous sections, $A_{\alpha}$ was just a background for the fermion fields. Here, we shall incorporate a Maxwell action so that $A_{\alpha}$ becomes dynamical. We then start from $Q E D_{4}$ 
with the fermionic field constrained to the region $0 \leqslant x_{3} \leqslant L$. The Abelian gauge field is, in principle, defined on an unconstrained region. However, its dynamics is determined entirely by the sources, which are, indeed, confined to $0 \leqslant x_{3} \leqslant L$. When the momenta involved in the processes are small in comparison with the scale $L^{-1}$, an effective theory can be obtained for the relevant degrees of freedom, which describe the dynamics on the plane $x_{3}=0$. This effective model contains Dirac fermions in interaction with a dynamical Abelian gauge field, and with an (also dynamical) scalar field, in $2+1$ dimensions. The spectrum of this theory also contains stripes: (Dirac) fermionic zero modes localized on linear defects.

Our starting point shall be to consider a model defined in terms of $S$, the action for massless $Q E D$ in four Euclidean dimensions

$$
S=S_{F}+S_{G}
$$

where $S_{F}$ and $S_{G}$ denote the fermionic and gauge field parts of the action, respectively. They are given by

$$
S_{F}=\int \mathrm{d}^{4} x \bar{\psi}(x) \not D \psi(x), \quad \not D=\not \partial+\mathrm{i} e \not A
$$

and

$$
S_{G}=\int \mathrm{d}^{4} x \frac{1}{4} F_{\alpha \beta} F_{\alpha \beta}, \quad F_{\alpha \beta}=\partial_{\alpha} A_{\beta}-\partial_{\beta} A_{\alpha} .
$$

We assume that the gauge field components, $A_{\alpha}$, are defined over the full four-dimensional Euclidean spacetime, so that the integration region in (41) is unbounded.

The fermionic field is, in turn, defined on a region of small width $L$ in the third spatial dimension, namely: $0 \leqslant x_{3} \leqslant L$, to simulate the physical situation of fermions in a quasi planar system. There are, of course, many different choices for the boundary conditions of the fermionic field at $x_{3}=0$ and $x_{3}=L$, all of them compatible with current conservation. In principle, the most natural one would be to impose the vanishing of the normal component of the current on the borders, namely, $j_{3}=0$ at $x_{3}=0, L$. Although this condition is natural, it is not an easy one to deal with from the point of view of the calculation, because it explicitly breaks translation invariance along $x_{3}$. To avoid this technical inconvenience, we prefer to use the simpler assumption that the fermionic field is $L$-periodic in $x_{3}$. This allows us to Fourier expand in that coordinate. From the point of view of the effective physics in the $0 \leqslant x_{3} \leqslant L$ region (the "dimensionally reduced" theory), both choices should lead to qualitatively similar results. The important point is, as we shall see, that the existence of a finite dimension allows for a description in terms of an infinite number of fermionic modes in the dimensionally reduced spacetime. The precise nature of the boundary conditions will of course affect the details of this phenomenon, like the spacing between the fermionic modes, but not the gross features and properties of the system.

The properties and objects we shall be concerned with can all be obtained, in principle, from the knowledge of $\mathcal{Z}[j ; \bar{\eta}, \eta]$, the generating functional of Green's functions 


$$
\mathcal{Z}[j ; \bar{\eta}, \eta]=\int \mathcal{D} A_{\mu} \mathcal{D} \bar{\psi} \mathcal{D} \psi \exp \left[-S+\int \mathrm{d}^{3} x \int_{0}^{L} \mathrm{~d} s\left(j_{\alpha} A_{\alpha}+\bar{\eta} \psi+\bar{\psi} \eta\right)\right]
$$

with $S$ as defined in (39). As we are interested exclusively in the phenomena localized on the $x_{3}=0$ plane, both the external source $j_{\alpha}$, and the fermionic current ie $\bar{\psi} \gamma_{\alpha} \psi$ are confined to the $0 \leqslant s \leqslant L$ region.

It is evident that, from the point of view of the description of the physics on the $x_{3}=0$ plane, the action for the gauge field yields more information than what is actually needed in our situation, since we are not going to consider processes with sources outside the region $0 \leqslant x_{3} \leqslant L$. It is possible, as we shall see, to obtain a $2+1$ dimensional action describing precisely the planar dynamics, and containing fermion and gauge fields with support on the $x_{3}=0$ plane. In a sense, we are going to "integrate out" gauge field modes, corresponding to excitations lying outside the plane.

As a first step, let us see how to pass to a description where the relevant gauge field dynamics is determined by an effective $2+1$ dimensional gauge field action. We may write $S_{G}^{J}$, the part of the action which involves the gauge field and its source, as follows:

$$
S_{G}^{J}[A, J]=S_{G}[A]+S_{g f}[A]+\int \mathrm{d}^{4} x J_{\alpha}(x) A_{\alpha}(x),
$$

where we have included a gauge fixing term, $S_{g f}[A]$, which, for the sake of simplicity, we take to be of the Feynman type. ${ }^{6}$

$$
S_{g f}=\int \mathrm{d}^{4} x \frac{1}{2}(\partial \cdot A)^{2} .
$$

$J_{\alpha}$ stands for the "full" current, namely, the external source for the gauge field $j_{\alpha}$ plus the fermionic current $i e \bar{\psi}(x) \gamma_{\alpha} \psi(x)$. We then integrate out the gauge field, obtaining a non-local current-current interaction:

$$
\int \mathcal{D} A_{\alpha} \exp \left\{-S_{G}^{J}[A, J]\right\}=\exp \left\{-S_{n l}[J]\right\},
$$

where

$$
S_{n l}[J]=-\frac{1}{2} \int \mathrm{d}^{4} x \mathrm{~d}^{4} y J_{\alpha}(x) K_{\alpha \beta}(x-y) J_{\beta}(y)
$$

with $K_{\alpha \beta}(x-y)$ denoting the (Feynman gauge) gauge field propagator:

$$
K_{\alpha \beta}(x-y)=\int \frac{\mathrm{d}^{4} k}{(2 \pi)^{4}} \mathrm{e}^{\mathrm{i} k \cdot(x-y)} \frac{\delta_{\alpha \beta}}{k^{2}} .
$$

Taking now into account the fact that the current $J_{\alpha}$ vanishes when either $x_{3}<0$ or $x_{3}>L$, we see that (46) may be written more precisely as follows:

$$
S_{n l}[J]=-\frac{1}{2} \int \mathrm{d}^{3} x \int_{0}^{L} \mathrm{~d} s_{1} \int \mathrm{d}^{3} y \int_{0}^{L} \mathrm{~d} s_{2} J_{\alpha}\left(x, s_{1}\right) K_{\alpha \beta}\left(x-y, s_{1}-s_{2}\right) J_{\beta}\left(y, s_{2}\right) .
$$

\footnotetext{
${ }^{6}$ The following derivation can, of course, also be implemented for different gauge fixings, but the calculations become more involved.
} 
In the $L \rightarrow 0$ limit, the current may be taken to be approximately $x_{3}$ independent, at least from the effective theory point of view, since a dependence on $x_{3}$ in this length scale would correspond to a momentum component comparable to the scale $L^{-1}$. Thus, we can replace in (48) $J_{\alpha}(x, s)$ by its average $\bar{J}_{\alpha}(x)$, defined by

$$
\bar{J}_{\alpha}(x)=\frac{1}{L} \int_{0}^{L} \mathrm{~d} s J_{\alpha}(x, s) .
$$

On the other hand, regarding the propagator, as $0 \leqslant s_{1,2} \leqslant L$, we may use the approximation

$$
K_{\alpha \beta}\left(x-y, s_{1}-s_{2}\right) \simeq \bar{K}_{\alpha \beta}(x-y)
$$

where

$$
\bar{K}_{\alpha \beta}(x-y)=K_{\alpha \beta}(x-y, 0) .
$$

Therefore, (46) reduces to a non local current-current interaction $\bar{S}_{n l}$ in $2+1$ dimensions

$$
\bar{S}_{n l}[\bar{J}]=-\frac{L^{2}}{2} \int \mathrm{d}^{3} x \int \mathrm{d}^{3} y \bar{J}_{\alpha}(x) \bar{K}_{\alpha \beta}(x-y) \bar{J}_{\beta}(y) .
$$

To obtain a more explicit form for $\bar{K}_{\alpha \beta}(x-y)$, we may write it in terms of its Fourier representation

$$
\bar{K}_{\alpha \beta}(x-y)=\int \frac{\mathrm{d}^{3} k}{(2 \pi)^{3}} \mathrm{e}^{\mathrm{i} k \cdot(x-y)} \int_{-\infty}^{+\infty} \frac{\mathrm{d} k_{3}}{2 \pi} \frac{\delta_{\alpha \beta}}{k^{2}} .
$$

Integrating over $k_{3}$, we easily see that the effective three-dimensional kernel for the currents $\bar{J}_{\mu}(x)$ is

$$
\bar{K}_{\mu \nu}(x-y)=\int \frac{\mathrm{d}^{3} k}{(2 \pi)^{3}} \mathrm{e}^{\mathrm{i} k \cdot(x-y)} \frac{\delta_{\mu \nu}}{\sqrt{k^{2}}}
$$

while for the $x_{3}$ component we have the scalar $\bar{K}_{s}$ :

$$
\bar{K}_{s}(x-y)=\int \frac{\mathrm{d}^{3} k}{(2 \pi)^{3}} \mathrm{e}^{\mathrm{i} k \cdot(x-y)} \frac{1}{\sqrt{k^{2}}} .
$$

Notice that from now on $k$ denotes the three dimensional momentum vector. $\bar{K}_{S}$ is treated separately, because $A_{3}$ behaves in fact as a scalar field under spacetime coordinate transformations in the $x_{3}=0$ hyperplane.

It is important to realize that the $2+1$ dimensional current $\bar{J}_{\mu}$ is conserved:

$$
\partial_{\mu} \bar{J}_{\mu}=0 \text {. }
$$

This follows from the fact that the current is approximately $x_{3}$-independent, in particular: $\partial_{3} J_{3}=0$, plus the usual continuity equation $\partial_{\alpha} J_{\alpha}=0$. Then we may actually replace $\delta_{\mu \nu}$ in (54) by its transverse part:

$$
\delta_{\mu \nu}^{\perp}=\delta_{\mu \nu}-\frac{k_{\mu} k_{v}}{k^{2}} .
$$


Summarizing, the result of integrating out the gauge field may, in the small- $L$ limit, be represented in terms of an effective $2+1$ dimensional action

$$
\begin{aligned}
\bar{S}_{n l}[\bar{J}]= & \frac{L^{2}}{2} \int \mathrm{d}^{3} x \int \mathrm{d}^{3} y \bar{J}_{\mu}(x) \bar{K}_{\mu \nu}^{\perp}(x-y) \bar{J}_{v}(y) \\
& +\frac{L^{2}}{2} \int \mathrm{d}^{3} x \int \mathrm{d}^{3} y \bar{J}_{s}(x) \bar{K}_{s}(x-y) \bar{J}_{S}(y)
\end{aligned}
$$

with $\bar{K}_{\mu \nu}^{\perp}$ as in (54), but with $\delta_{\mu \nu}^{\perp}$ instead of $\delta_{\mu \nu}$.

This effective action $\bar{S}_{n l}[\bar{J}]$ for the currents, can be equivalently rewritten as arising from the integration of a gauge field $a_{\mu}$, and a scalar field $\varphi$, both defined in the three dimensional space-time $x_{3}=0$. In other words, we can express the above action as the result of functionally integrating out (in $2+1$ dimensions) a gauge field $a_{\mu}$ and a scalar field $\varphi$, with the following action:

$$
\begin{aligned}
S_{n l}^{\prime}\left[\bar{J} ; a_{\mu}, \varphi\right]= & \frac{1}{2} \int \mathrm{d}^{3} x \int \mathrm{d}^{3} y a_{\mu}(x) \bar{K}_{\mu \nu}^{\perp-1}(x-y) a_{\nu}(y) \\
& +\frac{1}{2} \int \mathrm{d}^{3} x \int \mathrm{d}^{3} y \varphi(x) \bar{K}_{s}^{-1}(x-y) \varphi(y) \\
& +\int \mathrm{d}^{3} x \bar{J}_{\mu}^{\prime}(x) a_{\mu}(x)+\int \mathrm{d}^{3} x \bar{J}_{s}^{\prime}(x) \varphi(x),
\end{aligned}
$$

where $\bar{J}_{\alpha}^{\prime}(x)=L \bar{J}_{\alpha}(x)$. Note that the inclusion of this factor $L$ in the definition of the current reduces by one its mass dimensions, as it corresponds to the transition from fermionic fields in $3+1$ dimensions to $2+1$ dimensions. The bosonic fields, $a_{\mu}$ and $\varphi$, have the same mass dimension than their $3+1$ dimensional counterparts, because they are equipped with unusual, non-local kinetic terms.

Because of current conservation, we may also write a gauge invariant form for $S_{n l}^{\prime}\left[\bar{J} ; a_{\mu}, \varphi\right]$, by introducing the longitudinal part of $a_{\mu}$ into the game, obtaining:

$$
\begin{aligned}
S_{n l}^{\prime}\left[\bar{J} ; a_{\mu}, \varphi\right]= & \frac{1}{4} \int \mathrm{d}^{3} x F_{\mu \nu} \frac{1}{\sqrt{-\partial^{2}}} F_{\mu \nu}+\int \mathrm{d}^{3} x \bar{J}_{\mu}^{\prime}(x) a_{\mu}(x) \\
& +\int \mathrm{d}^{3} x \bar{J}_{s}^{\prime}(x) \varphi(x)+\frac{1}{2} \int \mathrm{d}^{3} x \partial_{\mu} \varphi \frac{1}{\sqrt{-\partial^{2}}} \partial_{\mu} \varphi
\end{aligned}
$$

where $F_{\mu \nu}=\partial_{\mu} a_{\nu}-\partial_{\nu} a_{\mu}$. This gauge invariant form should, of course, be gauge fixed in order to recover a regular action.

We have thus re-derived a known result: the electromagnetic interaction due to a Maxwell action, if restricted to charges living on a planar section of space, can be reproduced by introducing a non-local gauge invariant action in $2+1$ dimensions, like in $[11,12]$. This is of course different to the usual, Kaluza-Klein like "dimensional reduction" prescription and in fact is closer to an alternative prescription proposed in [14]. To understand the basic differences between the two prescriptions, let us concentrate in the case of a scalar field in $3+1$ dimensions with action

$$
S^{(3+1)}=\frac{1}{2} \int \mathrm{d}^{4} x \partial_{\mu} \varphi \partial^{\mu} \varphi
$$


with propagator $K^{3+1}$ satisfying in coordinate space the usual Green function equation

$$
-\partial^{2} K^{(3+1)}(x)=\delta(x)
$$

so that in coordinate and momentum (Minkowski) space one has

$$
\begin{aligned}
& K^{(3+1)}(x)=-\frac{4 \pi^{2} \mathrm{i}}{\left(x^{0}\right)^{2}-\left(x^{1}\right)^{2}-\left(x^{2}\right)^{2}-\left(x^{3}\right)^{2}-\mathrm{i} 0}, \\
& \tilde{K}^{(3+1)}(k)=-\frac{1}{\left(k_{0}\right)^{2}-\left(k_{1}\right)^{2}-\left(k_{2}\right)^{2}-\left(k_{3}\right)^{2}+\mathrm{i} 0} .
\end{aligned}
$$

The usual Kaluza-Klein dimensional reduction consists in this case in dropping $k_{3}^{2}$ in Eq. (64). Then, the propagator $K_{\mathrm{KK}}^{(2+1)}$ in the reduced space obeys the analogous to (62),

$$
-\partial^{2} K_{\mathrm{KK}}^{(2+1)}(x)=\delta(x)
$$

so that the reduced scalar theory corresponds to an action

$$
S_{\mathrm{KK}}^{(2+1)}=\frac{1}{2} \int \mathrm{d}^{3} x \partial_{\mu} \varphi \partial^{\mu} \varphi .
$$

The alternative prescription which leads to the reduction presented here corresponds to dropping $x^{3}$ in (63). To see this, note that if one puts $x_{3}=0$, the propagator $K^{(2+1)}$ does not satisfy anymore (65) but instead, as can be easily seen,

$$
\sqrt{-\partial^{2}} K^{(2+1)}(x)=\delta(x),
$$

so that the dynamics of the reduced scalar theory is governed by the action

$$
S^{(2+1)}=\frac{1}{2} \int \mathrm{d}^{3} x \partial_{\mu} \varphi \frac{1}{\sqrt{-\partial^{2}}} \partial^{\mu} \varphi,
$$

which is precisely the non-local action for the scalar field in (60). An analogous result, coinciding again with that in (60) can be found for a vector field like $a_{\mu}$.

Let us note that in the case in which the dimensional reductions is justified by the fact that the physical system is (quasi) planar, the alternative prescription which consists in dropping $x^{3}$, effectively compels the system to evolve in a plane keeping the nature of the interaction unchanged: for example, if in $3+1$ dimensions electrons interact via a $1 / r$ potential, they still interact through this potential in $2+1$ but with the third coordinate constrained to be zero. In contrast, the usual Kaluza-Klein prescription not only reduces the space but also changes drastically the nature of the interaction: in the example above, the electron interaction would change from $1 / r$ to $\log r$. If planar electrons are supposed to be subjected to $3+1$ interactions even when they are compelled to move in the plane, the alternative prescriptions then appears to be more physical than the usual Kaluza-Klein one.

The prize one pays when using the alternative prescription is the non-locality of the reduced action. It is interesting to note that, if looked at from the point of view of Minkowski spacetime, this non-locality is due to a branch cut singularity starting at zero momentum. Amusingly enough, this kind of singularity is entirely analogous to the one that appears when one considers the vacuum polarization function for massless fermions 
in $2+1$ dimensions. In that case, the branch cut singularity is of course due to the possibility of pair-creating fermions on-shell for any gauge field configuration with a nonzero momentum. The situation is, however, different to the "dimensional reduction" of our example, since the singularity here corresponds to modes that have been integrated out, and are in principle out of the spectrum of physical states. Thus the model is to be regarded as an open system, since the unitarity relations corresponding to a closed system would in turn require the introduction of asymptotic massless fermionic excitations into the game, like in the case of (unitary) massless $Q E D$ in $2+1$ dimensions.

The reduction of the fermionic part of the action proceeds as in Section 2, while the evaluation of the fermionic determinant has already been presented in Section 3.

\section{Discussion and conclusions}

So far we have obtained the effective action for the gauge fields (60), after reducing the original gauge action from $3+1$ to $2+1$ dimensions, and the one for the fermionic degrees of freedom (Eq. (38)), after integrating out the fermions. The final result is:

$$
\begin{aligned}
\mathcal{S}_{\text {eff }}\left[\varphi, a_{\mu}\right]= & \int \mathrm{d}^{3} x\left[\frac{4}{\pi^{2} L^{3}} \sum_{n=-\infty}^{\infty} \frac{1}{n^{4}} \cos (n e L \varphi)-\frac{14 \pi^{2}}{5 L^{3}}\right] \\
& +\frac{e^{2}}{12 \pi} \int \mathrm{d}^{3} x \sum_{n=-\infty}^{\infty} \frac{1}{\left|\omega_{n}+e \varphi\right|}\left[\left(\partial_{\mu} \varphi\right)^{2}+\left(F_{\mu \nu}\right)^{2}\right] \\
& +\frac{1}{4} \int \mathrm{d}^{3} x F_{\mu \nu} \frac{1}{\sqrt{-\partial^{2}}} F_{\mu \nu}+\frac{1}{2} \int \mathrm{d}^{3} x \partial_{\mu} \varphi \frac{1}{\sqrt{-\partial^{2}}} \partial_{\mu} \varphi .
\end{aligned}
$$

We see that the dynamics for the field that provides an "induced" mass for the fermions, $\varphi$, is given by a non-local interaction term, and a series of sine-Gordon like term. It is also through a series that is a functional of $\varphi$ that this last field couples to $a_{\mu}$.

As mentioned above, non-local effective theories for the electrodynamics of particles moving on a plane were discussed in [11-13]. In particular, a Lagrangian containing a nonlocal Maxwell term plus a coupling to matter current confined to a plane and coinciding with the first line in Lagrangian (60) was obtained in [12] where it was proven that this effective $2+1$ non-local Lagrangian was equivalent to one in which the current term is replaced by a Chern-Simons term. This last Lagrangian describes precisely the bosonized version of a $2+1$ massless fermion theory [11] and, as shown in [13], this is not a coincidence. Indeed, the functional approach to bosonization $[15,16]$ for $2+1$ free Dirac fermions with mass $m$, leads to a bosonization recipe which is exact for the matter current:

$$
\bar{J}^{\mu}(x) \rightarrow \frac{1}{\sqrt{4 \pi}} \varepsilon^{\mu \nu \alpha} \partial_{\nu} a_{\alpha} .
$$

Concerning the bosonic action, it cannot be written explicitly, except for certain particular limits. In particular, in the vanishing mass limit one can compute exactly the bosonic action [13] obtaining a result which coincides with the first line in Eq. (60) (if one uses (70) to bosonize the matter current). It is worth emphasizing here that we have not started from a 
$2+1$ model but rather from massless $Q E D_{4}$, and that the reduction mechanism explained above brings into play, besides the usual $a_{\mu}$ field, also an scalar field $\varphi$ which has a nontrivial, sine-Gordon like dynamics and it is moreover coupled to $a_{\mu}$. The necessity for the existence of this field may be traced back of course to the fact that the compactified dimension allows for a certain non-trivial structure in that direction, reflected in the large gauge transformations of which the scalar field is the subject.

To finish this discussion we look at the propagation of fermions in a $\left(a_{\mu}, \varphi\right)$ background. In a first approximation, the dynamics of these background fields could be regarded as entirely determined by the effective action (69) only, and as a next step one should introduce the reaction of the fermionic fields on the bosons. Looking back at the action (18) for the fermions in $2+1$, we see that for each species $\chi_{n}^{(1)}$ and $\chi_{n}^{(2)}$, there will be a fermionic zero mode every time $\omega_{n}+e \varphi$ passes through zero, i.e., when $e \varphi= \pm 2 n \pi / L$. These zero modes are produced because of the Callan and Harvey mechanism, since there is a change of sign for the mass term of a $2+1$ dimensional fermion. Since we have two 2-component fermions, and their mass terms have opposite signs, there shall be two chiral fermions, of opposite chiralities, localized around each defect. This is of course equivalent to saying that we shall have a massless Dirac fermionic zero mode around each defect. The existence of these gapless zero modes has of course relevance for the calculation of transport properties.

If we restrict ourselves to configurations having an approximate invariance under translations along one of the spatial coordinates, the effective action for the $\varphi$ field will of course be the one of a two dimensional field theory with a potential periodic in the field, and with an unusual kinetic term. Despite this last property, the periodic nature of the potential implies that there is room for the existence of a non-trivial topological charge $Q$, defined as for the case of the sine-Gordon model. If the system is invariant under translations in $x_{2}$, then

$$
Q=\frac{e L}{2 \pi} \int_{-\infty}^{+\infty} \mathrm{d} x_{1} \partial_{1} \varphi(x)=\frac{e L}{2 \pi}[\varphi(+\infty)-\varphi(-\infty)] .
$$

A configuration of definite charge $Q=q$, corresponds to the field $\varphi$ starting in one of the minima at $x_{1}=-\infty$ and covering $q$ periods of the potential before finishing at another minimum of the potential, at $x_{1}=+\infty$. We may assume, for the sake of clarity, that the effective potential is identical to the first component of the series. Then, there will be exactly $q$ fermionic zero modes, since they appear each time the potential passes through a maximum. This discussion shows that there is an interesting relation between the topological solitonic charge and the number of fermionic zero modes.

\section{Acknowledgements}

C.D.F. and A.L. are supported by CONICET and Instituto Balseiro, Argentina. This work is supported in part by grants from ANPCYT (PICT 97/0053, 97/2285 and 98/0303924), CONICET, and by Fundación Antorchas, Argentina. 


\section{References}

[1] R. Rajaraman, Solitons and Instantons, North Holland, Amsterdam, 1982.

[2] C.D. Fosco, A. López, Nucl. Phys. B 538 (1999) 685.

[3] C. Callan, J.A. Harvey, Nucl. Phys. B 250 (1985) 427.

[4] I.J.R. Aitchison, C.M. Fraser, Phys. Rev. D 31 (1985) 2605.

[5] G. Grignani, G. Semenoff, P. Sodano, Phys. Rev. D 53 (1996) 7157.

[6] J. Kapusta, Finite-Temperature Field Theory, Cambridge Univ. Press, 1993, Appendix A.3, and references therein.

[7] G. Grignani, G. Semenoff, P. Sodano, O. Tirkkonen, Nucl. Phys. B 473 (1996) 143.

[8] C.D. Fosco, G.L. Rossini, F.A. Schaposnik, Phys. Rev. Lett. 79 (1997) 1980; Phys. Rev. D 56 (1997) 6547; Phys. Rev. D 59 (1999) 085012.

[9] C.D. Fosco, E. Fradkin, A. López, Phys. Lett. B 451 (1999) 31.

[10] A.R. Kavalov, I.K. Kostov, A.K. Sedrakyan, Phys. Lett. B 175 (1986) 331.

[11] E.C. Marino, Phys. Lett. B 263 (1991) 634.

[12] E.C. Marino, Nucl. Phys. B 408 (1993) 551.

[13] D.G. Barci, C.D. Fosco, L.E. Oxman, Phys. Lett. B 375 (1996) 267.

[14] J.D. Edelstein, C. Núñez, F.A. Schaposnik, J.J. Giambiagi, Mod. Phys. Lett. A 11 (1995) 1037.

[15] E. Fradkin, F.A. Schaposnik, Phys. Lett. B 338 (1994) 254.

[16] F.A. Schaposnik, Phys. Lett. B 356 (1995) 39. 